Honam Mathematical J. 35 (2013), No. 1, pp. 051-071

http://dx.doi.org/10.5831/HMJ.2013.35.1.51

\title{
RELATIONSHIPS BETWEEN INTEGRAL TRANSFORMS AND CONVOLUTIONS ON AN ANALOGUE OF WIENER SPACE*
}

Dong Hyun Cho

\begin{abstract}
In the present paper, we evaluate the analytic conditional Fourier-Feynman transforms and convolution products of unbounded function which is the product of the cylinder function and the function in a Banach algebra which is defined on an analogue of Wiener space and useful in the Feynman integration theories and quantum mechanics. We then investigate the inverse transforms of the function with their relationships and finally prove that the analytic conditional Fourier-Feynman transforms of the conditional convolution products for the functions, can be expressed in terms of the product of the conditional Fourier-Feynman transforms of each function.
\end{abstract}

\section{Introduction and preliminaries}

Let $C_{0}[0, t]$ denote the Wiener space, that is, the space of real-valued continuous paths $x$ on the closed interval $[0, t]$ with $x(0)=0$. On the space $C_{0}[0, t]$, Chang and Skoug [2] introduced the concepts of conditional Fourier-Feynman transform and conditional convolution product and then, examined the effects that drift has on the conditional Fourier-Feynman transform, the conditional convolution product, and various relationships that occur between them. Moreover, on $C[0, t]$, the space of real-valued continuous paths on $[0, t]$, Kim [13] extended the relationships between the conditional convolution product and the

Received January 13, 2013. Accepted January 28, 2013.

2010 Mathematics Subject Classification. 28C20, 44A35, 60G15, 60H05.

Key words and phrases. analogue of Wiener space, analytic conditional Feynman integral, analytic conditional Fourier-Feynman transform, conditional convolution product, conditional Wiener integral, Wiener space.

*This research was supported by Basic Science Research Program through the National Research Foundation(NRF) of Korea funded by the Ministry of Education, Science and Technology(2012-0002477). 
$L_{p}(1 \leq p \leq \infty)$-analytic conditional Fourier-Feynman transform of the functions in a Banach algebra which corresponds to the CameronStorvick's Banach algebra $\mathcal{S}[1]$. The author and his coauthors $[3,4,5$, $6,7,14]$ also established relationships between them for various functions on $C[0, t]$. In particular, he [3] derived an evaluation formula for the $L_{p}$-analytic conditional Fourier-Feynman transforms and convolution products of unbounded functions with the conditioning functions $X_{n}$ and $X_{n+1}$ on $C[0, t]$ given by $X_{n}(x)=\left(x\left(t_{0}\right), x\left(t_{1}\right), \cdots, x\left(t_{n}\right)\right)$ and $X_{n+1}(x)=\left(x\left(t_{0}\right), x\left(t_{1}\right), \cdots, x\left(t_{n}\right), x\left(t_{n+1}\right)\right)$, where $n$ is a positive integer and $0=t_{0}<t_{1}<\cdots<t_{n}<t_{n+1}=t$ is a partition of $[0, t]$, and then, derived their relationships. Note that $X_{n}$ is independent of the present positions of the paths in $C[0, t]$ while $X_{n+1}$ wholly depends on the present positions.

In this paper, when $n=0$, we further develop the relationships on the space $\left(C[0, t], w_{\varphi}\right)$, an analogue of Wiener space associated with a probability measure $\varphi$ on the Borel class $\mathcal{B}(\mathbb{R})$ of $\mathbb{R}[11,15,16]$. In fact, using simple formulas for the conditional expectations given $X_{0}$ and $X_{1}$, we proceed to evaluate the $L_{p}$-analytic conditional Fourier-Feynman transforms and convolution products for the functions of the form

$$
f_{r}\left(\left(v_{1}, x\right), \cdots,\left(v_{r}, x\right)\right) \int_{L_{2}[0, t]} \exp \{i(v, x)\} d \sigma(v)
$$

for $w_{\varphi}$-a.e. $x \in C[0, t]$, where $f_{r} \in L_{p}\left(\mathbb{R}^{r}\right),\left\{v_{1}, \cdots, v_{r}\right\}$ is an orthonormal set in $L_{2}[0, t]$ and $\sigma$ is a complex Borel measure of bounded variation on $L_{2}[0, t]$. We then investigate the inverse transforms and the relationships between them of the functions given by (1.1). Finally, we show that the $L_{p}$-analytic conditional Fourier-Feynman transform of the conditional convolution product for the functions $\Psi_{1}$ and $\Psi_{2}$ given by (1.1), can be expressed by the formula

$$
\begin{aligned}
& T_{q}^{(p)}\left[\left[\left(\Psi_{1} * \Psi_{2}\right)_{q} \mid X_{0}\right]\left(\cdot, \xi_{0}\right) \mid X_{0}\right]\left(y, \zeta_{0}\right) \\
= & {\left[T_{q}^{(p)}\left[\Psi_{1} \mid X_{0}\right]\left(\frac{1}{\sqrt{2}} y, 0\right)\right]\left[T_{q}^{(p)}\left[\Psi_{2} \mid X_{0}\right]\left(\frac{1}{\sqrt{2}} y, 0\right)\right] }
\end{aligned}
$$

for a nonzero real $q, w_{\varphi}$-a.e. $y \in C[0, t]$ and $P_{X_{0}}$-a.e. $\xi_{0}, \zeta_{0} \in \mathbb{R}$. Thus the analytic conditional Fourier-Feynman transforms of the conditional convolution products for the functions, can be interpreted as the product of the analytic conditional Fourier-Feynman transforms of each function. Note that they are independent of the initial positions of paths in $C[0, t]$ while they depend only on the present time. 
Let $\mathbb{C}, \mathbb{C}_{+}$and $\mathbb{C}_{+}^{\sim}$ denote the sets of complex numbers, complex numbers with positive real parts and nonzero complex numbers with nonnegative real parts, respectively. Let $C[0, t]$ be the space of all real-valued continuous functions on the closed interval $[0, t]$ with its Borel class $\mathcal{B}(C[0, t])$. For a probability measure $\varphi$ on $(\mathbb{R}, \mathcal{B}(\mathbb{R}))$, let $w_{\varphi}$ be an analogue of the Wiener measure on $\mathcal{B}(C[0, t])$ associated with $\varphi[11,15,16]$. Let $F: C[0, t] \rightarrow \mathbb{C}$ be integrable and $X$ be a random vector on $C[0, t]$ assuming that the value space of $X$ is a normed space equipped with the Borel $\sigma$-algebra. Then, we have the conditional expectation $E[F \mid X]$ of $F$ given $X$ from a well known probability theory. Furthermore, there exists a $P_{X}$-integrable $\mathbb{C}$-valued function $\psi$ on the value space of $X$ such that $E[F \mid X](x)=(\psi \circ X)(x)$ for $w_{\varphi}$-a.e. $x \in C[0, t]$, where $P_{X}$ is the probability distribution of $X$. The function $\psi$ is called the conditional $w_{\varphi}$-integral of $F$ given $X$ and it is also denoted by $E[F \mid X]$.

Throughout this paper, let $X_{0}: C[0, t] \rightarrow \mathbb{R}$ and $X_{1}: C[0, t] \rightarrow \mathbb{R}^{2}$ be given by $X_{0}(x)=x(0)$ and $X_{1}(x)=(x(0), x(t))$. Let $F: C[0, t] \rightarrow \mathbb{C}$ be a function such that $F\left(\lambda^{-\frac{1}{2}} \cdot\right)$ is integrable for $\lambda>0$. Then we have the following formula from $[9]$

$$
\begin{aligned}
& E\left[F\left(\lambda^{-\frac{1}{2}} \cdot\right) \mid X_{1}\left(\lambda^{-\frac{1}{2}} \cdot\right)\right]\left(\xi_{0}, \xi_{1}\right) \\
= & E\left[F\left(\lambda^{-\frac{1}{2}}\left(x-x(0)-\frac{\dot{t}}{t}(x(t)-x(0))\right)+\xi_{0}+\dot{\dot{t}}\left(\xi_{1}-\xi_{0}\right)\right)\right]
\end{aligned}
$$

for $P_{X_{1}^{\lambda} \text {-a.e. }}\left(\xi_{0}, \xi_{1}\right) \in \mathbb{R}^{2}$, where $P_{X_{1}^{\lambda}}$ is the probability distribution of $X_{1}\left(\lambda^{-\frac{1}{2}} \cdot\right)$ on $\left(\mathbb{R}^{2}, \mathcal{B}\left(\mathbb{R}^{2}\right)\right)$. For $y \in C[0, t]$, let

$$
I_{F}^{\lambda}\left(y, \xi_{0}, \xi_{1}\right)=E\left[F\left(\lambda^{-\frac{1}{2}}\left(x-x(0)-\frac{\dot{t}}{t}(x(t)-x(0))\right)+y+\xi_{0}+\dot{\dot{t}}\left(\xi_{1}-\xi_{0}\right)\right)\right] \text {, }
$$

where the expectation is taken over the variable $x$. Moreover, we have from $[8]$

$E\left[F\left(\lambda^{-\frac{1}{2}} \cdot\right) \mid X_{0}\left(\lambda^{-\frac{1}{2}} \cdot\right)\right]\left(\xi_{0}\right)=\left(\frac{\lambda}{2 \pi t}\right)^{\frac{1}{2}} \int_{\mathbb{R}} I_{F}^{\lambda}\left(0, \xi_{0}, \xi_{1}\right) \exp \left\{-\frac{\lambda\left(\xi_{1}-\xi_{0}\right)^{2}}{2 t}\right\} d \xi_{1}$

for $P_{X_{0}^{\lambda}}$-a.e. $\xi_{0} \in \mathbb{R}$, where $P_{X_{0}^{\lambda}}$ is the probability distribution of $X_{0}\left(\lambda^{-\frac{1}{2}} \cdot\right)$ on $(\mathbb{R}, \mathcal{B}(\mathbb{R}))$. For $y \in C[0, t]$, let

$$
K_{F}^{\lambda}\left(y, \xi_{0}\right)=\left(\frac{\lambda}{2 \pi t}\right)^{\frac{1}{2}} \int_{\mathbb{R}} I_{F}^{\lambda}\left(y, \xi_{0}, \xi_{1}\right) \exp \left\{-\frac{\lambda\left(\xi_{1}-\xi_{0}\right)^{2}}{2 t}\right\} d \xi_{1} .
$$

If $I_{F}^{\lambda}\left(0, \xi_{0}, \xi_{1}\right)$ has the analytic extension $J_{\lambda}^{*}(F)\left(\xi_{0}, \xi_{1}\right)$ on $\mathbb{C}_{+}$as a function of $\lambda$, then it is called the analytic conditional Wiener $w_{\varphi}$-integral 
of $F$ given $X_{1}$ with parameter $\lambda$ and denoted by $E^{a n w_{\lambda}}\left[F \mid X_{1}\right]\left(\xi_{0}, \xi_{1}\right)=$ $J_{\lambda}^{*}(F)\left(\xi_{0}, \xi_{1}\right)$ for $\left(\xi_{0}, \xi_{1}\right) \in \mathbb{R}^{2}$. Moreover, if for a nonzero real $q, E^{a n w_{\lambda}}[F$ $\left.\mid X_{1}\right]\left(\xi_{0}, \xi_{1}\right)$ has the limit as $\lambda$ approaches to $-i q$ through $\mathbb{C}_{+}$, then it is called the conditional analytic Feynman $w_{\varphi}$-integral of $F$ given $X_{1}$ with parameter $q$ and denoted by

$$
E^{a n f_{q}}\left[F \mid X_{1}\right]\left(\xi_{0}, \xi_{1}\right)=\lim _{\lambda \rightarrow-i q} E^{a n w_{\lambda}}\left[F \mid X_{1}\right]\left(\xi_{0}, \xi_{1}\right) .
$$

Similarly, the definitions of $E^{a n w_{\lambda}}\left[F \mid X_{0}\right]\left(\xi_{0}\right)$ and $E^{a n f_{q}}\left[F \mid X_{0}\right]\left(\xi_{0}\right)$ are understood with $K_{F}^{\lambda}\left(0, \xi_{0}\right)$ if $X_{1}$ is replaced by $X_{0}$.

For a given extended real number $p$ with $1<p \leq \infty$, suppose that $p$ and $p^{\prime}$ are related by $\frac{1}{p}+\frac{1}{p^{\prime}}=1$ (possibly $p^{\prime}=1$ if $p=\infty$ ). Let $F_{n}$ and $F$ be measurable functions such that $\lim _{n \rightarrow \infty} \int_{C[0, t]} \mid F_{n}(x)-$ $\left.F(x)\right|^{p^{\prime}} d w_{\varphi}(x)=0$. Then we write l.i.m. ${ }_{n \rightarrow \infty}\left(w^{p^{\prime}}\right)\left(F_{n}\right)=F$ and call $F$ the limit in the mean of order $p^{\prime}$. A similar definition is understood when $n$ is replaced by a continuously varying parameter.

Let $F$ and $G$ be defined on $C[0, t]$. For $\lambda \in \mathbb{C}_{+}$and $w_{\varphi}$-a.e. $y \in C[0, t]$, let

$$
T_{\lambda}\left[F \mid X_{1}\right]\left(y, \xi_{0}, \xi_{1}\right)=E^{a n w_{\lambda}}\left[F(y+\cdot) \mid X_{1}\right]\left(\xi_{0}, \xi_{1}\right)
$$

for $P_{X_{1}}$-a.e. $\left(\xi_{0}, \xi_{1}\right) \in \mathbb{R}^{2}$ if it exists. For a nonzero real $q$ and $w_{\varphi^{-}}$ a.e. $y \in C[0, t]$, define the $L_{1}$-analytic conditional Fourier-Feynman transform $T_{q}^{(1)}\left[F \mid X_{1}\right]$ of $F$ given $X_{1}$ by the formula

$$
T_{q}^{(1)}\left[F \mid X_{1}\right]\left(y, \xi_{0}, \xi_{1}\right)=E^{a n f_{q}}\left[F(y+\cdot) \mid X_{1}\right]\left(\xi_{0}, \xi_{1}\right)
$$

for $P_{X_{1}}$-a.e. $\left(\xi_{0}, \xi_{1}\right) \in \mathbb{R}^{2}$ if it exists. For $1<p \leq \infty$, define the $L_{p^{-}}$ analytic conditional Fourier-Feynman transform $T_{q}^{(p)}\left[F \mid X_{1}\right]$ of $F$ given $X_{1}$ by the formula

$$
T_{q}^{(p)}\left[F \mid X_{1}\right]\left(\cdot, \xi_{0}, \xi_{1}\right)=\operatorname{li.m.~}_{\lambda \rightarrow-i q}\left(w^{p^{\prime}}\right)\left(T_{\lambda}\left[F \mid X_{1}\right]\left(\cdot, \xi_{0}, \xi_{1}\right)\right)
$$

for $P_{X_{1}}$-a.e. $\left(\xi_{0}, \xi_{1}\right) \in \mathbb{R}^{2}$, where $\lambda$ approaches to $-i q$ through $\mathbb{C}_{+}$. We also define the conditional convolution product $\left[(F * G)_{\lambda} \mid X_{1}\right]$ of $F$ and $G$ given $X_{1}$ by the formula, for $w_{\varphi}$-a.e. $y \in C[0, t]$

$$
\begin{aligned}
& {\left[(F * G)_{\lambda} \mid X_{1}\right]\left(y, \xi_{0}, \xi_{1}\right)} \\
& = \begin{cases}E^{a n w_{\lambda}}\left[F\left(\frac{y+\cdot}{\sqrt{2}}\right) G\left(\frac{y-\cdot}{\sqrt{2}}\right) \mid X_{1}\right]\left(\xi_{0}, \xi_{1}\right), & \lambda \in \mathbb{C}_{+} ; \\
E^{a n f_{q}}\left[F\left(\frac{y+\cdot}{\sqrt{2}}\right) G\left(\frac{y-\cdot}{\sqrt{2}}\right) \mid X_{1}\right]\left(\xi_{0}, \xi_{1}\right), \quad \lambda=-i q\end{cases}
\end{aligned}
$$


if they exist for $P_{X_{1}}$-a.e. $\left(\xi_{0}, \xi_{1}\right) \in \mathbb{R}^{2}$. If $\lambda=-i q$, we replace $[(F *$ $\left.G)_{\lambda} \mid X_{1}\right]$ by $\left[(F * G)_{q} \mid X_{1}\right]$. Similar definitions and notations are understood with $\xi_{0} \in \mathbb{R}$ if $X_{1}$ is replaced by $X_{0}$.

\section{The initial position-dependent conditional transform and convolution}

Let $\|\cdot\|_{2}$ denote the $L_{2}$-norm on the real Hilbert space $L_{2}[0, t]$. For $v$ in $L_{2}[0, t]$ and $x$ in $C[0, t]$, let $(v, x)$ denote the Paley-Wiener-Zygmund integral of $v$ according to $x$ [11]. Note that by $\langle\cdot, \cdot\rangle_{\mathbb{R}^{r}}$, the dot product on the $r$-dimensional Euclidean space $\mathbb{R}^{r}$, is also denoted.

Throughout this paper, let $\left\{v_{l}: l=1, \cdots, r\right\}$ be an orthonormal subset of $L_{2}[0, t]$ such that $\mathcal{G} \equiv\left\{v_{l}-\frac{1}{t} \int_{0}^{t} v_{l}(s) d s: l=1, \cdots, r\right\}$ is an independent set. Let $\left\{e_{l}: l=1, \cdots, r\right\}$ be the orthonormal set obtained from $\mathcal{G}$ by the Gram-Schmidt orthonormalization process. Now, for $l=$ $1, \cdots, r$, let $v_{l}-\frac{1}{t} \int_{0}^{t} v_{l}(s) d s=\sum_{j=1}^{r} \beta_{l j} e_{j}$ be the linear combinations of the $e_{j}$ s and let $A=\left[\beta_{l j}\right]_{r \times r}$ be the coefficient matrix of the combinations. Define the linear transformation $T_{A}: \mathbb{R}^{r} \rightarrow \mathbb{R}^{r}$ by $T_{A} \vec{z}=\vec{z} A^{T}$ where $A^{T}$ is the transpose of $A$ and $\vec{z}$ is any row-vector in $\mathbb{R}^{r}$. Note that $A$ is invertible so that $T_{A}$ is an isomorphism. For $v \in L_{2}[0, t]$, let

$$
c_{l}(v)=\int_{0}^{t} v(s) e_{l}(s) d s-\frac{1}{t} \int_{0}^{t} v(s) d s \int_{0}^{t} e_{l}(s) d s
$$

for $l=1, \cdots, r$ and let $(\vec{v}, x)=\left(\left(v_{1}, x\right), \cdots,\left(v_{r}, x\right)\right)$ for $x \in C[0, t]$. Furthermore, for $\lambda \in \mathbb{C}_{+}^{\sim}, v \in L_{2}[0, t]$ and $\vec{u}=\left(u_{1}, \cdots, u_{r}\right) \in \mathbb{R}^{r}$, let

$$
A_{1}(\lambda, v, \vec{u})=\exp \left\{\frac{1}{2 \lambda}\left[\sum_{j=1}^{r}\left[\lambda i u_{j}+c_{j}(v)\right]^{2}-\left\|v-\frac{1}{t} \int_{0}^{t} v(s) d s\right\|_{2}^{2}\right]\right\}
$$

Let $\vec{V}_{t}=\frac{1}{t}\left(\int_{0}^{t} v_{1}(s) d s, \cdots, \int_{0}^{t} v_{r}(s) d s\right)$. For $\vec{z} \in \mathbb{R}^{r}$ and $\left(\xi_{0}, \xi_{1}\right) \in \mathbb{R}^{2}$, let

$$
\begin{aligned}
A_{2}\left(f, g ; \lambda, \vec{z}, \xi_{0}, \xi_{1}, v\right) & =\left(\frac{\lambda}{2 \pi}\right)^{\frac{r}{2}} \int_{\mathbb{R}^{r}} f\left(\vec{z}+\left(\xi_{1}-\xi_{0}\right) \vec{V}_{t}+T_{A} \vec{u}\right) \\
& \times g\left(\vec{z}-\left(\xi_{1}-\xi_{0}\right) \vec{V}_{t}-T_{A} \vec{u}\right) A_{1}(\lambda, v, \vec{u}) d \vec{u}
\end{aligned}
$$

if exists, where $f$ and $g$ are Borel measurable on $\mathbb{R}^{r}$. Note that by the Bessel's inequality,

$$
\left|A_{1}(\lambda, v, \vec{u})\right| \leq \exp \left\{-\frac{\operatorname{Re} \lambda}{2}\|\vec{u}\|_{\mathbb{R}^{r}}^{2}\right\} \leq 1 .
$$


Using the same method as used in the proof of Theorem 3.1 in [10], we can prove the following lemma.

Lemma 2.1. Let $f$ be Borel measurable on $\mathbb{R}^{r}$. Then, for $\lambda>0$ and $v \in L_{2}[0, t]$,

$$
\begin{aligned}
& \int_{C[0, t]} f\left(\lambda^{-\frac{1}{2}}\left(\vec{v}, x-x(0)-\frac{\dot{t}}{t}(x(t)-x(0))\right)\right) \\
& \times \exp \left\{i \lambda^{-\frac{1}{2}}\left(v, x-x(0)-\frac{\dot{t}}{t}(x(t)-x(0))\right)\right\} d w_{\varphi}(x) \\
\stackrel{*}{=} & A_{2}(f, 1 ; \lambda, \overrightarrow{0}, 0,0, v),
\end{aligned}
$$

where $\stackrel{*}{=}$ means that if either side exists, then both sides exist and they are equal.

For $1 \leq p \leq \infty$, let $\mathcal{A}_{r}^{(p)}$ be the space of the cylinder functions $F_{r}$ of the form given by

$$
F_{r}(x)=f_{r}(\vec{v}, x)
$$

for $w_{\varphi}$-a.e. $x \in C[0, t]$, where $f_{r} \in L_{p}\left(\mathbb{R}^{r}\right)$. Note that, without loss of generality, we can take $f_{r}$ to be Borel measurable. Let $\mathcal{M}=\mathcal{M}\left(L_{2}[0, t]\right)$ be the class of all $\mathbb{C}$-valued Borel measures of bounded variation over $L_{2}[0, t]$ and let $\mathcal{S}_{w_{\varphi}}$ be the space of all functions $F$ which for $\sigma \in \mathcal{M}$ have the form

$$
F(x)=\int_{L_{2}[0, t]} \exp \{i(v, x)\} d \sigma(v)
$$

for $w_{\varphi}$-a.e. $x \in C[0, t]$. Note that $\mathcal{S}_{w_{\varphi}}$ is a Banach algebra which is equivalent to $\mathcal{M}$ with the norm $\|F\|=\|\sigma\|$, the total variation of $\sigma$ [11].

Using the same method as used in the proof of Theorems 2.2, 2.3 and 2.4 of [3], we have the following theorems.

Theorem 2.2. Let $1 \leq p \leq \infty$. For $w_{\varphi}$-a.e. $x \in C[0, t]$, let $\Psi(x)=$ $F(x) F_{r}(x)$, where $F_{r} \in \mathcal{A}_{r}^{(p)}$ and $F \in \mathcal{S}_{w_{\varphi}}$ are given by (2.5) and (2.6), respectively. Then for $\lambda \in \mathbb{C}_{+}, w_{\varphi}$-a.e. $y \in C[0, t]$ and $P_{X_{1}}$-a.e. $\left(\xi_{0}, \xi_{1}\right) \in$ $\mathbb{R}^{2}$,

$$
\begin{aligned}
& T_{\lambda}\left[\Psi \mid X_{1}\right]\left(y, \xi_{0}, \xi_{1}\right) \\
& =\int_{L_{2}[0, t]} H_{1}\left(y, \xi_{0}, \xi_{1}, v, v\right) A_{2}\left(f_{r}, 1 ; \lambda,(\vec{v}, y), \xi_{0}, \xi_{1}, v\right) d \sigma(v)
\end{aligned}
$$


where $A_{2}$ is given by (2.3) and $H_{1}$ is given by

$$
H_{1}\left(y, \xi_{0}, \xi_{1}, v_{1}, v_{2}\right)=\exp \left\{i\left[\left(v_{1}, y\right)+\frac{\xi_{1}-\xi_{0}}{t} \int_{0}^{t} v_{2}(s) d s\right]\right\}
$$

for $v_{1}, v_{2} \in L_{2}[0, t]$. Moreover, as a function of $y, T_{\lambda}\left[\Psi \mid X_{1}\right]\left(\cdot, \xi_{0}, \xi_{1}\right) \in$ $L_{p}(C[0, t])$. If $p=1$, then for nonzero real $q, T_{q}^{(1)}\left[\Psi \mid X_{1}\right]\left(y, \xi_{0}, \xi_{1}\right)$ is given by (2.7) replacing $\lambda$ by $-i q$ and $T_{q}^{(1)}\left[\Psi \mid X_{1}\right]\left(\cdot, \xi_{0}, \xi_{1}\right) \in L_{\infty}(C[0, t])$.

Theorem 2.3. Let the assumptions be as given in Theorem 2.2 with one exception $1 \leq p \leq 2$ and let $\frac{1}{p}+\frac{1}{p^{\prime}}=1$. Then for $\lambda \in \mathbb{C}_{+}$and $P_{X_{1}}$-a.e. $\left(\xi_{0}, \xi_{1}\right) \in \mathbb{R}^{2}, T_{\lambda}\left[\Psi \mid X_{1}\right]\left(\cdot, \xi_{0}, \xi_{1}\right) \in L_{p^{\prime}}(C[0, t])$. Furthermore, suppose that $\sigma$ is concentrated on the subspace of constant functions in $L_{2}[0, t]$. Then for a nonzero real $q, w_{\varphi}$-a.e. $y \in C[0, t]$,

$$
\begin{aligned}
T_{q}^{(p)}\left[\Psi \mid X_{1}\right]\left(y, \xi_{0}, \xi_{1}\right)= & \left(f_{r}\left(T_{A} \cdot\right) * \Phi(-i q, \cdot)\right)\left(\left(\left(\xi_{1}-\xi_{0}\right) \vec{V}_{t}+(\vec{v}, y)\right)\left(A^{T}\right)^{-1}\right) \\
& \times \int_{L_{2}[0, t]} \exp \left\{i(v, y)+i\left(\xi_{1}-\xi_{0}\right) v\right\} d \sigma(v),
\end{aligned}
$$

where for $\lambda \in \mathbb{C}_{+}^{\sim}$ and $\vec{u} \in \mathbb{R}^{r}$

$$
\Phi(\lambda, \vec{u})=\left(\frac{\lambda}{2 \pi}\right)^{\frac{1}{2}} \exp \left\{-\frac{\lambda}{2}\|\vec{u}\|_{\mathbb{R}^{r}}^{2}\right\} .
$$

In this case, $T_{q}^{(p)}\left[\Psi \mid X_{1}\right]\left(\cdot, \xi_{0}, \xi_{1}\right) \in L_{p^{\prime}}(C[0, t])$.

Theorem 2.4. Let $F_{r} \in \mathcal{A}_{r}^{\left(p_{1}\right)}, G_{r} \in \mathcal{A}_{r}^{\left(p_{2}\right)}$ and $f_{r}, g_{r}$ be related by (2.5), respectively, where $1 \leq p_{1}, p_{2} \leq \infty$. Let $F_{1}, F_{2} \in \mathcal{S}_{w_{\varphi}}$ and $\sigma_{1}, \sigma_{2} \in \mathcal{M}\left(L_{2}[0, t]\right)$ be related by (2.6), respectively. Furthermore, let $\frac{1}{p_{1}}+\frac{1}{p_{1}^{\prime}}=1, \frac{1}{p_{2}}+\frac{1}{p_{2}^{\prime}}=1, \Psi_{1}(x)=F_{r}(x) F_{1}(x)$ and $\Psi_{2}(x)=G_{r}(x) F_{2}(x)$ for $w_{\varphi}$-a.e. $x \in C[0, t]$. Then for $\lambda \in \mathbb{C}_{+}, w_{\varphi}$-a.e. $y \in C[0, t]$ and $P_{X_{1}}$-a.e. $\left(\xi_{0}, \xi_{1}\right) \in \mathbb{R}^{2}$,

$$
\begin{aligned}
& {\left[\left(\Psi_{1} * \Psi_{2}\right)_{\lambda} \mid X_{1}\right]\left(y, \xi_{0}, \xi_{1}\right) } \\
= & \int_{L_{2}[0, t]} \int_{L_{2}[0, t]} H_{1}\left(\frac{1}{\sqrt{2}} y, \frac{1}{\sqrt{2}} \xi_{0}, \frac{1}{\sqrt{2}} \xi_{1}, v_{1}+v_{2}, v_{1}-v_{2}\right) A_{2}\left(f_{r}, g_{r} ;\right. \\
& \left.2 \lambda,\left(\vec{v}, \frac{1}{\sqrt{2}} y\right), \frac{1}{\sqrt{2}} \xi_{0}, \frac{1}{\sqrt{2}} \xi_{1}, v_{1}-v_{2}\right) d \sigma_{1}\left(v_{1}\right) d \sigma_{2}\left(v_{2}\right)
\end{aligned}
$$

where $A_{2}$ and $H_{1}$ are given by (2.3) and (2.8), respectively. Furthermore, as a function of $y,\left[\left(\Psi_{1} * \Psi_{2}\right)_{\lambda} \mid X_{1}\right]\left(\cdot, \xi_{0}, \xi_{1}\right) \in L_{1}(C[0, t])$ if either $p_{2} \leq$ $p_{1}^{\prime}$ or $p_{1} \leq p_{2}^{\prime},\left[\left(\Psi_{1} * \Psi_{2}\right)_{\lambda} \mid X_{1}\right]\left(\cdot, \xi_{0}, \xi_{1}\right) \in L_{p_{2}}(C[0, t])$ if $p_{2} \geq p_{1}^{\prime}$ and $\left[\left(\Psi_{1} * \Psi_{2}\right)_{\lambda} \mid X_{1}\right]\left(\cdot, \xi_{0}, \xi_{1}\right) \in L_{p_{1}}(C[0, t])$ if $p_{1} \geq p_{2}^{\prime}$. 


\section{The initial position-independent conditional transform and convolution}

In this section, we evaluate the initial position-independent conditional Fourier-Feynman transform and conditional convolution product of the functions as given in the previous section.

Let $\Gamma_{t}=\frac{1}{1+t\left\|T_{A}^{-1} \vec{V}_{t}\right\|_{\mathbb{R}^{r}}^{2}}$. For $\lambda \in \mathbb{C}_{+}^{\sim}, v \in L_{2}[0, t]$ and $\vec{u} \in \mathbb{R}^{r}$, let

$$
\begin{aligned}
& B_{1}(\lambda, v, \vec{u}) \\
= & \exp \left\{\frac{t \Gamma_{t}}{2 \lambda}\left[i\left[\frac{1}{t} \int_{0}^{t} v(s) d s-\left\langle\vec{c}(v), T_{A}^{-1} \vec{V}_{t}\right\rangle_{\mathbb{R}^{r}}\right]+\lambda\left\langle T_{A}^{-1} \vec{V}_{t}, \vec{u}\right\rangle_{\mathbb{R}^{r}}\right]^{2}\right\}
\end{aligned}
$$

where $\vec{c}=\left(c_{1}, \cdots, c_{r}\right)$ and the $c_{j}$ s are given by (2.1). Furthermore, for $\vec{z} \in \mathbb{R}^{r}$ let

$$
\begin{aligned}
& B_{2}(f, g ; \lambda, \vec{z}, v) \\
= & \Gamma_{t}^{\frac{1}{2}}\left(\frac{\lambda}{2 \pi}\right)^{\frac{r}{2}} \int_{\mathbb{R}^{r}} f\left(\vec{z}+T_{A} \vec{u}\right) g\left(\vec{z}-T_{A} \vec{u}\right) A_{1}(\lambda, v, \vec{u}) B_{1}(\lambda, v, \vec{u}) d \vec{u}
\end{aligned}
$$

if exists, where $f$ and $g$ are Borel measurable on $\mathbb{R}^{r}$ and $A_{1}$ is given by (2.2). Using the following well-known integration formula

$$
\int_{\mathbb{R}} \exp \left\{-a u^{2}+i b u\right\} d u=\left(\frac{\pi}{a}\right)^{\frac{1}{2}} \exp \left\{-\frac{b^{2}}{4 a}\right\}
$$

for $a \in \mathbb{C}_{+}$and $b \in \mathbb{R}$, we can prove the following lemma from Theorem 3.4 of $[10]$.

Lemma 3.1. Let $f$ be Borel measurable on $\mathbb{R}^{r}$ and let $A_{2}$ be given by (2.3). Then for $\lambda>0, \xi_{0} \in \mathbb{R}$ and $v \in L_{2}[0, t]$

$$
\begin{aligned}
& \left(\frac{\lambda}{2 \pi t}\right)^{\frac{1}{2}} \int_{\mathbb{R}} \exp \left\{\frac{i\left(\xi_{1}-\xi_{0}\right)}{t} \int_{0}^{t} v(s) d s\right\} A_{2}\left(f, 1 ; \lambda, \overrightarrow{0}, \xi_{0}, \xi_{1}, v\right) \\
& \times \exp \left\{-\frac{\lambda\left(\xi_{1}-\xi_{0}\right)^{2}}{2 t}\right\} d \xi_{1} \stackrel{*}{=} B_{2}(f, 1 ; \lambda, \overrightarrow{0}, v)
\end{aligned}
$$

where $B_{2}$ is given by (3.2).

Theorem 3.2. Under the assumptions as given in Theorem 2.2, we have for $\lambda \in \mathbb{C}_{+}$, w $w_{\varphi}$-a.e. $y \in C[0, t]$ and $P_{X_{0}}$-a.e. $\xi_{0} \in \mathbb{R}$

$$
T_{\lambda}\left[\Psi \mid X_{0}\right]\left(y, \xi_{0}\right)=\int_{L_{2}[0, t]} B_{2}\left(f_{r}, 1 ; \lambda,(\vec{v}, y), v\right) \exp \{i(v, y)\} d \sigma(v)
$$


where $B_{2}$ is given by (3.2). Moreover, as a function of $y, T_{\lambda}\left[\Psi \mid X_{0}\right]\left(\cdot, \xi_{0}\right) \in$ $L_{p}(C[0, t])$. If $p=1$, then for nonzero real $q, T_{q}^{(1)}\left[\Psi \mid X_{0}\right]\left(y, \xi_{0}\right)$ is given by (3.4) replacing $\lambda$ by $-i q$ and $T_{q}^{(1)}\left[\Psi \mid X_{0}\right]\left(\cdot, \xi_{0}\right) \in L_{\infty}(C[0, t])$.

Proof. For $\lambda>0, y \in C[0, t]$ and $\xi_{0} \in \mathbb{R}$,

$$
\begin{array}{r}
K_{\Psi}^{\lambda}\left(y, \xi_{0}\right)=\left(\frac{\lambda}{2 \pi t}\right)^{\frac{1}{2}} \int_{L_{2}[0, t]} \exp \{i(v, y)\} \int_{\mathbb{R}} \exp \left\{\frac{i\left(\xi_{1}-\xi_{0}\right)}{t} \int_{0}^{t} v(s) d s\right\} \\
\times A_{2}\left(f_{r}((\vec{v}, y)+\cdot), 1 ; \lambda, \overrightarrow{0}, \xi_{0}, \xi_{1}, v\right) \exp \left\{-\frac{\lambda\left(\xi_{1}-\xi_{0}\right)^{2}}{2 t}\right\} d \xi_{1} d \sigma(v)
\end{array}
$$

by Theorem 2.2. By Lemma 3.1,

$$
K_{\Psi}^{\lambda}\left(y, \xi_{0}\right) \stackrel{*}{=} \int_{L_{2}[0, t]} B_{2}\left(f_{r}, 1 ; \lambda,(\vec{v}, y), v\right) \exp \{i(v, y)\} d \sigma(v) .
$$

By (2.4), the Bessel's inequality and the Schwarz's inequality, we have for $\lambda \in \mathbb{C}_{+}^{\sim}$

$$
\left|A_{1}(\lambda, v, \vec{u}) B_{1}(\lambda, v, \vec{u})\right| \leq \exp \left\{-\frac{\Gamma_{t} \operatorname{Re} \lambda}{2}\|\vec{u}\|_{\mathbb{R}^{r}}^{2}\right\} \leq 1
$$

from the same method as used in the proof of Theorem 3.4 of [10]. Note that by the change of variable theorem

$$
\left\|f\left(\vec{z}+T_{A} \cdot\right)\right\|_{p}^{p}=\left|\operatorname{det}\left(A^{T}\right)^{-1}\right|\left\|f_{r}\right\|_{p}^{p}<\infty .
$$

By the Hölder's inequality, the first inequality of (3.5), the Morera's theorem and the dominated convergence theorem, we have the equality in the theorem as the analytic extension of $K_{\Psi}^{\lambda}\left(y, \xi_{0}\right)$ on $\mathbb{C}_{+}$. We also have by (3.5), (3.6), Theorem 3.5 of [11] and the Young's inequality

$$
\begin{aligned}
& \int_{C[0, t]}\left|T_{\lambda}\left[\Psi \mid X_{0}\right]\left(y, \xi_{0}\right)\right|^{p} d w_{\varphi}(y) \\
\leq & |\operatorname{det}(A)|\|\sigma\|^{p}\left(\frac{|\lambda|}{\Gamma_{t} \operatorname{Re} \lambda}\right)^{\frac{p r}{2}}\left\|\left|f_{r}\left(T_{A} \cdot\right)\right| * \Phi\left(\Gamma_{t} \operatorname{Re} \lambda, \cdot\right)\right\|_{p}^{p} \\
< & \infty
\end{aligned}
$$

if $1 \leq p<\infty$, where $\Phi$ is given by $(2.9)$, and $\left\|T_{\lambda}\left[\Psi \mid X_{0}\right]\left(\cdot, \xi_{0}\right)\right\|_{\infty} \leq$ $\|\sigma\|\left\|f_{r}\right\|_{\infty}\left(\frac{|\lambda|}{\Gamma_{t} \operatorname{Re} \lambda}\right)^{\frac{r}{2}}$ if $p=\infty$. If $p=1$, the final result follows from the second inequality of (3.5) and the dominated convergence theorem.

Theorem 3.3. Let the assumptions be as given in Theorem 3.2 with one exception $1 \leq p \leq 2$ and let $\frac{1}{p}+\frac{1}{p^{\prime}}=1$. Then for $\lambda \in \mathbb{C}_{+}$and $P_{X_{0}}$-a.e. $\xi_{0} \in \mathbb{R}, T_{\lambda}\left[\Psi \mid X_{0}\right]\left(\cdot, \xi_{0}\right) \in L_{p^{\prime}}(C[0, t])$. Furthermore, suppose 
that $\sigma$ is concentrated on the subspace of constant functions in $L_{2}[0, t]$ and $\int_{0}^{t} v_{l}(s) d s=0$ for $l=1, \cdots, r$. Then for a nonzero real $q, w_{\varphi}$-a.e. $y \in C[0, t]$ and $P_{X_{0}}$-a.e. $\xi_{0} \in \mathbb{R}$,

$T_{q}^{(p)}\left[\Psi \mid X_{0}\right]\left(y, \xi_{0}\right)=\left(f_{r} * \Phi(-i q, \cdot)\right)(\vec{v}, y) \int_{L_{2}[0, t]} D_{-i q}(v) \exp \{i(v, y)\} d \sigma(v)$,

where $\Phi$ is given by (2.9), $D_{\lambda}(v)=\exp \left\{-\frac{t}{2 \lambda} v^{2}\right\}$ for $\lambda \in \mathbb{C}_{+}^{\sim}$. In this case, $T_{q}^{(p)}\left[\Psi \mid X_{0}\right]\left(\cdot, \xi_{0}\right) \in L_{p^{\prime}}(C[0, t])$.

Proof. Let $1<p \leq 2$. Using the same process as used in the proof of Theorem 3.2, we have for $\lambda \in \mathbb{C}_{+}$,

$$
\begin{aligned}
& \int_{C[0, t]}\left|T_{\lambda}\left[\Psi \mid X_{0}\right]\left(y, \xi_{0}\right)\right|^{p^{\prime}} d w_{\varphi}(y) \\
\leq & |\operatorname{det}(A)|\|\sigma\|^{p^{\prime}}\left(\frac{|\lambda|}{\Gamma_{t} \operatorname{Re} \lambda}\right)^{\frac{p^{\prime} r}{2}}\left\|\left|f_{r}\left(T_{A} \cdot\right)\right| * \Phi\left(\Gamma_{t} \operatorname{Re} \lambda, \cdot\right)\right\|_{p^{\prime}}^{p^{\prime}} \\
< & \infty
\end{aligned}
$$

where the last inequality follows from Lemma 1.1 of [12]. Now, suppose that $\sigma$ is concentrated on the subspace of constant functions in $L_{2}[0, t]$ and $\int_{0}^{t} v_{l}(s) d s=0$ for $l=1, \cdots, r$. Then $\vec{V}_{t}=\overrightarrow{0} \in \mathbb{R}^{r}, \Gamma_{t}=1$ and $A$ is the identity matrix so that we have for $\sigma$-a.e $v \in L_{2}[0, t], \lambda \in \mathbb{C}_{+}^{\sim}$ and $\vec{u} \in \mathbb{R}^{r}$

$$
\left(\frac{\lambda}{2 \pi}\right)^{\frac{r}{2}} A_{1}(\lambda, v, \vec{u})=\Phi(\lambda, \vec{u}) \text { and } B_{1}(\lambda, v, \vec{u})=D_{\lambda}(v)
$$

By Theorem 3.2 and (3.7), we have for $\lambda \in \mathbb{C}_{+}^{\sim}$, $w_{\varphi}$-a.e. $y \in C[0, t]$ and $P_{X_{0}}$-a.e. $\xi_{0} \in \mathbb{R}$

$$
\begin{aligned}
T_{\lambda}\left[\Psi \mid X_{0}\right]\left(y, \xi_{0}\right) & =\int_{L_{2}[0, t]} B_{2}\left(f_{r}, 1 ; \lambda,(\vec{v}, y), v\right) \exp \{i(v, y)\} d \sigma(v) \\
& =\left(f_{r} * \Phi(\lambda, \cdot)\right)(\vec{v}, y) \int_{L_{2}[0, t]} D_{\lambda}(v) \exp \{i(v, y)\} d \sigma(v) .
\end{aligned}
$$

By Theorem 3.5 of [11] and Lemma 1.1 of [12]

$$
\int_{C[0, t]}\left|T_{q}^{(p)}\left[\Psi \mid X_{0}\right]\left(y, \xi_{0}\right)\right|^{p^{\prime}} d w_{\varphi}(y) \leq\|\sigma\|^{p^{\prime}}\left\|f_{r} * \Phi(-i q, \cdot)\right\|_{p^{\prime}}^{p^{\prime}}<\infty .
$$


By Theorem 3.5 of [11], the Minkowski inequality and the triangle inequality, we also have for $\lambda \in \mathbb{C}_{+}$

$$
\begin{aligned}
& \left\|T_{\lambda}\left[\Psi \mid X_{0}\right]\left(\cdot, \xi_{0}\right)-T_{q}^{(p)}\left[\Psi \mid X_{0}\right]\left(\cdot, \xi_{0}\right)\right\|_{p^{\prime}} \\
\leq & {\left[\int _ { \mathbb { R } ^ { r } } \left[\int_{L_{2}[0, t]}\left|D_{\lambda}(v)\left(f_{r} * \Phi(\lambda, \cdot)\right)(\vec{u})-D_{-i q}(v)\left(f_{r} * \Phi(-i q, \cdot)\right)(\vec{u})\right|\right.\right.} \\
& \left.d|\sigma|(v)]^{p^{\prime}} d \vec{u}\right]^{\frac{1}{p^{\prime}}} \\
\leq & {\left[\int _ { \mathbb { R } ^ { r } } \left[\left|\left(f_{r} * \Phi(\lambda, \cdot)\right)(\vec{u})-\left(f_{r} * \Phi(-i q, \cdot)\right)(\vec{u})\right| \int_{L_{2}[0, t]}\left|D_{\lambda}(v)\right| d|\sigma|(v)\right.\right.} \\
& \left.\left.+\left|\left(f_{r} * \Phi(-i q, \cdot)\right)(\vec{u})\right| \int_{L_{2}[0, t]}\left|D_{\lambda}(v)-D_{-i q}(v)\right| d|\sigma|(v)\right]^{p^{\prime}} d \vec{u}\right]^{\frac{1}{p^{\prime}}} \\
\leq & \|\sigma\|\left\|f_{r} * \Phi(\lambda, \cdot)-f_{r} * \Phi(-i q, \cdot)\right\|_{p^{\prime}}+\left\|f_{r} * \Phi(-i q, \cdot)\right\|_{p^{\prime}} \int_{L_{2}[0, t]} \\
& \left|D_{\lambda}(v)-D_{-i q}(v)\right| d|\sigma|(v)
\end{aligned}
$$

which converges to 0 as $\lambda$ approaches to $-i q$ through $\mathbb{C}_{+}$by the dominated convergence theorem and Lemma 1.2 of [12]. If $p=1$, the conclusions follow from Theorem 3.2. Now, the proof is completed.

Remark 3.4. The orthonormal subset $\left\{v_{l}: l=1, \cdots, r\right\}$ of $L_{2}[0, t]$ satisfying $\int_{0}^{t} v_{l}(s) d s=0$ for $l=1, \cdots, r$ exists [10].

Theorem 3.5. Under the assumptions as given in Theorem 2.4, we have for $\lambda \in \mathbb{C}_{+}$, w $w_{\varphi}$-a.e. $y \in C[0, t]$ and $P_{X_{0}}$-a.e. $\xi_{0} \in \mathbb{R}$

$$
\begin{aligned}
{\left[\left(\Psi_{1} * \Psi_{2}\right)_{\lambda} \mid X_{0}\right]\left(y, \xi_{0}\right) } & =\int_{L_{2}[0, t]} \int_{L_{2}[0, t]} \exp \left\{i\left(v_{1}+v_{2}, \frac{1}{\sqrt{2}} y\right)\right\} \\
\times & B_{2}\left(f_{r}, g_{r} ; 2 \lambda,\left(\vec{v}, \frac{1}{\sqrt{2}} y\right), v_{1}-v_{2}\right) d \sigma_{1}\left(v_{1}\right) d \sigma_{2}\left(v_{2}\right)
\end{aligned}
$$

where $B_{2}$ is given by (3.2). Furthermore, as a function of $y,\left[\left(\Psi_{1} *\right.\right.$ $\left.\left.\Psi_{2}\right)_{\lambda} \mid X_{0}\right]\left(\cdot, \xi_{0}\right) \in L_{1}(C[0, t])$ if either $p_{2} \leq p_{1}^{\prime}$ or $p_{1} \leq p_{2}^{\prime},\left[\left(\Psi_{1} * \Psi_{2}\right)_{\lambda} \mid X_{1}\right]$ $\left(\cdot, \xi_{0}\right) \in L_{p_{2}}(C[0, t])$ if $p_{2} \geq p_{1}^{\prime}$ and $\left[\left(\Psi_{1} * \Psi_{2}\right)_{\lambda} \mid X_{0}\right]\left(\cdot, \xi_{0}\right) \in L_{p_{1}}(C[0, t])$ if $p_{1} \geq p_{2}^{\prime}$. 
Proof. For $\xi_{0} \in \mathbb{R}, \lambda>0$ and $y \in C[0, t]$, we have by Theorem 2.4

$$
\begin{aligned}
& {\left[\left(\Psi_{1} * \Psi_{2}\right)_{\lambda} \mid X_{0}\right]\left(y, \xi_{0}\right) } \\
= & \left(\frac{\lambda}{2 \pi t}\right)^{\frac{1}{2}} \int_{L_{2}[0, t]} \int_{L_{2}[0, t]} \int_{\mathbb{R}} H_{1}\left(\frac{1}{\sqrt{2}} y, \frac{1}{\sqrt{2}} \xi_{0}, \frac{1}{\sqrt{2}} \xi_{1}, v_{1}+v_{2}, v_{1}-v_{2}\right) \\
& \times A_{2}\left(f_{r}, g_{r} ; 2 \lambda,\left(\vec{v}, \frac{1}{\sqrt{2}} y\right), \frac{1}{\sqrt{2}} \xi_{0}, \frac{1}{\sqrt{2}} \xi_{1}, v_{1}-v_{2}\right) \\
& \times \exp \left\{-\frac{\lambda\left(\xi_{1}-\xi_{0}\right)^{2}}{2 t}\right\} d \xi_{1} d \sigma_{1}\left(v_{1}\right) d \sigma_{2}\left(v_{2}\right) .
\end{aligned}
$$

Note that by the change of variable theorem,

$$
\begin{aligned}
& A_{2}\left(f_{r}, g_{r} ; 2 \lambda,\left(\vec{v}, \frac{1}{\sqrt{2}} y\right), \frac{1}{\sqrt{2}} \xi_{0}, \frac{1}{\sqrt{2}} \xi_{1}, v_{1}-v_{2}\right) \\
= & A_{2}\left(h_{r}, 1 ; \lambda, \overrightarrow{0}, \xi_{0}, \xi_{1}, \frac{1}{\sqrt{2}}\left(v_{1}-v_{2}\right)\right)
\end{aligned}
$$

where $h_{r}(\vec{z})=f_{r}\left(\frac{1}{\sqrt{2}}[(\vec{v}, y)+\vec{z}]\right) g_{r}\left(\frac{1}{\sqrt{2}}[(\vec{v}, y)-\vec{z}]\right)$ for $\vec{z} \in \mathbb{R}^{r}$. Then by Lemma 3.1

$$
\begin{aligned}
& {\left[\left(\Psi_{1} * \Psi_{2}\right)_{\lambda} \mid X_{0}\right]\left(y, \xi_{0}\right) } \\
= & \left(\frac{\lambda}{2 \pi t}\right)^{\frac{1}{2}} \int_{L_{2}[0, t]} \int_{L_{2}[0, t]} \exp \left\{\frac{i}{\sqrt{2}}\left(v_{1}+v_{2}, y\right)\right\} \int_{\mathbb{R}} \exp \left\{\frac{i\left(\xi_{1}-\xi_{0}\right)}{\sqrt{2} t}\right. \\
& \left.\times \int_{0}^{t}\left(v_{1}(s)-v_{2}(s)\right) d s\right\} A_{2}\left(h_{r}, 1 ; \lambda, \overrightarrow{0}, \xi_{0}, \xi_{1}, \frac{1}{\sqrt{2}}\left(v_{1}-v_{2}\right)\right) \\
& \times \exp \left\{-\frac{\lambda\left(\xi_{1}-\xi_{0}\right)^{2}}{2 t}\right\} d \xi_{1} d \sigma_{1}\left(v_{1}\right) d \sigma_{2}\left(v_{2}\right) \\
= & \int_{L_{2}[0, t]} \int_{L_{2}[0, t]} \exp \left\{i\left(v_{1}+v_{2}, \frac{1}{\sqrt{2}} y\right)\right\} B_{2}\left(f_{r}, g_{r} ; 2 \lambda,\left(\vec{v}, \frac{1}{\sqrt{2}} y\right),\right. \\
& \left.v_{1}-v_{2}\right) d \sigma_{1}\left(v_{1}\right) d \sigma_{2}\left(v_{2}\right) .
\end{aligned}
$$

Thus for $\lambda \in \mathbb{C}_{+}$, we have by (3.5), Theorem 3.5 of [11] and the change of variable theorem

$$
\begin{aligned}
& \int_{C[0, t]}\left|\left[\left(\Psi_{1} * \Psi_{2}\right)_{\lambda} \mid X_{0}\right]\left(y, \xi_{0}\right)\right| d w_{\varphi}(y) \\
\leq & \left\|\sigma_{1}\right\|\left\|\sigma_{2}\right\|\left(\frac{|\lambda|}{\Gamma_{t} \operatorname{Re} \lambda}\right)^{\frac{r}{2}} \int_{\mathbb{R}^{r}} \int_{\mathbb{R}^{r}}\left|f_{r}\left(\frac{1}{\sqrt{2}}\left(\vec{u}+T_{A} \vec{z}\right)\right)\right|\left|g_{r}\left(\frac{1}{\sqrt{2}}\left(\vec{u}-T_{A} \vec{z}\right)\right)\right| \\
& \times \Phi\left(\Gamma_{t} \operatorname{Re} \lambda, \vec{z}\right) d \vec{z} d \vec{u}
\end{aligned}
$$


where $\Phi$ is given by (2.9). By the same method as used in the proof of Theorem 3.1 of [5], we have the theorem.

\section{Relationships between the conditional transforms and con- volutions}

In this section, we investigate the inverse transforms of the conditional Fourier-Feynman transforms of the functions as given in the previous sections. We also show that the analytic conditional Fourier-Feynman transforms of the conditional convolution product for the functions, can be expressed as the product of the analytic conditional Fourier-Feynman transforms of each function as desired.

In the following two theorems, we derive the inverse transforms of the conditional Fourier-Feynman transforms of the function $\Psi$ as given in the previous sections.

Theorem 4.1. For $\sigma \in \mathcal{M}, y \in C[0, t],\left(\xi_{0}, \xi_{1}\right),\left(\zeta_{0}, \zeta_{1}\right) \in \mathbb{R}^{2}$ and $f_{r}: \mathbb{R}^{r} \rightarrow \mathbb{C}$, let

$$
\begin{aligned}
\Psi_{\xi_{0}, \xi_{1}, \zeta_{0}, \zeta_{1}}(y)= & f_{r}\left((\vec{v}, y)+\left(\zeta_{1}-\zeta_{0}+\xi_{1}-\xi_{0}\right) \vec{V}_{t}\right) \\
& \times \int_{L_{2}[0, t]} \exp \left\{i(v, y)+i\left(\zeta_{1}-\zeta_{0}+\xi_{1}-\xi_{0}\right) v\right\} d \sigma(v) .
\end{aligned}
$$

Then, under the assumptions as given in Theorem 2.2 with one exception that $\sigma$ is concentrated on the subspace of constant functions in $L_{2}[0, t]$, we have for $P_{X_{1}}$-a.e. $\left(\xi_{0}, \xi_{1}\right),\left(\zeta_{0}, \zeta_{1}\right) \in \mathbb{R}^{2}$

$$
\left\|T_{\bar{\lambda}}\left[T_{\lambda}\left[\Psi \mid X_{1}\right]\left(\cdot, \xi_{0}, \xi_{1}\right) \mid X_{1}\right]\left(\cdot, \zeta_{0}, \zeta_{1}\right)-\Psi_{\xi_{0}, \xi_{1}, \zeta_{0}, \zeta_{1}}\right\|_{p} \longrightarrow 0
$$

for $1 \leq p<\infty$, and for $1 \leq p \leq \infty$

$$
T_{\bar{\lambda}}\left[T_{\lambda}\left[\Psi \mid X_{1}\right]\left(\cdot, \xi_{0}, \xi_{1}\right) \mid X_{1}\right]\left(\cdot, \zeta_{0}, \zeta_{1}\right) \longrightarrow \Psi_{\xi_{0}, \xi_{1}, \zeta_{0}, \zeta_{1}}(y)
$$

for $w_{\varphi}$-a.e. $y \in C[0, t]$, as $\lambda$ approaches to $-i q$ through $\mathbb{C}_{+}$.

Proof. For $\lambda_{1}>0, \lambda \in \mathbb{C}_{+}, w_{\varphi}$-a.e. $y \in C[0, t]$ and $P_{X_{1}}$-a.e. $\left(\xi_{0}, \xi_{1}\right)$, $\left(\zeta_{0}, \zeta_{1}\right) \in \mathbb{R}^{2}$, we have by Lemma 2.1 and Theorem 2.2

$$
\begin{aligned}
& I_{T_{\lambda}\left[\Psi \mid X_{1}\right]\left(\cdot, \xi_{0}, \xi_{1}\right)}^{\lambda_{1}}\left(y, \zeta_{0}, \zeta_{1}\right) \\
= & \int_{L_{2}[0, t]} \exp \left\{i(v, y)+i\left(\zeta_{1}-\zeta_{0}+\xi_{1}-\xi_{0}\right) v\right\} \int_{C[0, t]} \exp \left\{i \lambda_{1}^{-\frac{1}{2}}(v, x-\right. \\
& \left.\left.x(0)-\frac{\dot{t}}{t}(x(t)-x(0))\right)\right\} \int_{\mathbb{R}^{r}} f_{r}\left(\lambda_{1}^{-\frac{1}{2}}(\vec{v}, x-x(0)-\dot{\bar{t}}(x(t)-x(0)))\right. \\
& \left.+(\vec{v}, y)+\left(\zeta_{1}-\zeta_{0}+\xi_{1}-\xi_{0}\right) \vec{V}_{t}+T_{A} \vec{u}\right) \Phi(\lambda, \vec{u}) d \vec{u} d w_{\varphi}(x) d \sigma(v)
\end{aligned}
$$




$$
\begin{aligned}
= & \int_{L_{2}[0, t]} \exp \left\{i(v, y)+i\left(\zeta_{1}-\zeta_{0}+\xi_{1}-\xi_{0}\right) v\right\} \int_{\mathbb{R}^{r}} \int_{\mathbb{R}^{r}} f_{r}((\vec{v}, y)+ \\
& \left.\left(\zeta_{1}-\zeta_{0}+\xi_{1}-\xi_{0}\right) \vec{V}_{t}+T_{A}(\vec{z}+\vec{u})\right) \Phi(\lambda, \vec{u}) \Phi\left(\lambda_{1}, \vec{z}\right) d \vec{z} d \vec{u} d \sigma(v)
\end{aligned}
$$

where $\Phi$ is given by (2.9). By the analytic continuation, we have the existence of

$$
T_{\lambda_{1}}\left[T_{\lambda}\left[\Psi \mid X_{1}\right]\left(\cdot, \xi_{0}, \xi_{1}\right) \mid X_{1}\right]\left(y, \zeta_{0}, \zeta_{1}\right)
$$

for $\lambda_{1} \in \mathbb{C}_{+}$. Let $\vec{l}=\vec{z}+\vec{u}$. By the change of variable theorem and (3.3)

$$
\begin{aligned}
& T_{\bar{\lambda}}\left[T_{\lambda}\left[\Psi \mid X_{1}\right]\left(\cdot, \xi_{0}, \xi_{1}\right) \mid X_{1}\right]\left(\cdot, \zeta_{0}, \zeta_{1}\right) \\
= & \left(\frac{|\lambda|}{2 \pi}\right)^{r} \int_{L_{2}[0, t]} \exp \left\{i(v, y)+i\left(\zeta_{1}-\zeta_{0}+\xi_{1}-\xi_{0}\right) v\right\} \int_{\mathbb{R}^{r}} \int_{\mathbb{R}^{r}} f_{r}((\vec{v}, y) \\
& \left.+\left(\zeta_{1}-\zeta_{0}+\xi_{1}-\xi_{0}\right) \vec{V}_{t}+T_{A} \vec{l}\right) \exp \left\{-\operatorname{Re} \lambda\|\vec{u}\|_{\mathbb{R}^{r}}^{2}+\bar{\lambda}\left\langle\vec{u}, \vec{l}_{\mathbb{R}^{r}}-\frac{\lambda}{2}\|\vec{l}\|_{\mathbb{R}^{r}}^{2}\right\}\right. \\
& d \vec{u} d \vec{l} d \sigma(v) \\
= & \left(\frac{|\lambda|^{2}}{4 \pi \operatorname{Re} \lambda}\right)^{\frac{r}{2}} \int_{L_{2}[0, t]} \exp \left\{i(v, y)+i\left(\zeta_{1}-\zeta_{0}+\xi_{1}-\xi_{0}\right) v\right\} \int_{\mathbb{R}^{r}} f_{r}\left(T_{A}(((\vec{v}, y)\right. \\
& \left.\left.\left.+\left(\zeta_{1}-\zeta_{0}+\xi_{1}-\xi_{0}\right) \vec{V}_{t}\right)\left(A^{T}\right)^{-1}-\vec{l}\right)\right) \exp \left\{-\frac{|\lambda|^{2}}{4 \operatorname{Re} \lambda}\|\vec{l}\|_{\mathbb{R}^{r}}^{2}\right\} d \vec{l} d \sigma(v) \\
= & \epsilon^{-r}\left(f_{r}\left(T_{A} \cdot\right) * \Phi(1, \cdot / \epsilon)\right)\left(\left((\vec{v}, y)+\left(\zeta_{1}-\zeta_{0}+\xi_{1}-\xi_{0}\right) \vec{V}_{t}\right)\left(A^{T}\right)^{-1}\right) \\
& \times \int_{L_{2}[0, t]} \exp \left\{i(v, y)+i\left(\zeta_{1}-\zeta_{0}+\xi_{1}-\xi_{0}\right) v\right\} d \sigma(v)
\end{aligned}
$$

where $\epsilon=\left(2 \operatorname{Re} \lambda /|\lambda|^{2}\right)^{1 / 2}>0$. By the same method as used in the proof of Theorem 4.1 of [3], we have the theorem.

Theorem 4.2. Under the assumptions as given in Theorem 3.2 with exceptions that $\sigma$ is concentrated on the subspace of constant functions in $L_{2}[0, t]$ and $\int_{0}^{t} v_{l}(s) d s=0$ for $l=1, \cdots, r$, we have for $P_{X_{0}}$-a.e. $\xi_{0}, \zeta_{0} \in \mathbb{R}$

$$
\left\|T_{\bar{\lambda}}\left[T_{\lambda}\left[\Psi \mid X_{0}\right]\left(\cdot, \xi_{0}\right) \mid X_{0}\right]\left(\cdot, \zeta_{0}\right)-\Psi\right\|_{p} \longrightarrow 0
$$

for $1 \leq p<\infty$, and for $1 \leq p \leq \infty$

$$
T_{\bar{\lambda}}\left[T_{\lambda}\left[\Psi \mid X_{0}\right]\left(\cdot, \xi_{0}\right) \mid X_{0}\right]\left(y, \zeta_{0}\right) \longrightarrow \Psi(y)
$$

for $w_{\varphi}$-a.e. $y \in C[0, t]$, as $\lambda$ approaches to $-i q$ through $\mathbb{C}_{+}$.

Proof. Note that $\vec{V}_{t}=\overrightarrow{0}, \Gamma_{t}=1$ and $T_{A}$ is the identity transformation on $\mathbb{R}^{r}$. For $\lambda \in \mathbb{C}_{+}, \lambda_{1}>0, y \in C[0, t], \xi_{0} \in \mathbb{R}$ and $\left(\zeta_{0}, \zeta_{1}\right) \in \mathbb{R}^{2}$, we 
have

$$
\begin{aligned}
& I_{T_{\lambda}\left[\Psi \mid X_{0}\right]\left(\cdot, \xi_{0}\right)}^{\lambda_{1}}\left(y, \zeta_{0}, \zeta_{1}\right) \\
= & \int_{L_{2}[0, t]} \exp \left\{i(v, y)+i\left(\zeta_{1}-\zeta_{0}\right) v\right\} \int_{\mathbb{R}^{r}} \Phi(\lambda, \vec{u}) D_{\lambda}(v) \int_{C[0, t]} f_{r}\left(\lambda_{1}^{-\frac{1}{2}}(\vec{v}, x-\right. \\
& \left.\left.x(0)-\frac{\dot{t}}{t}(x(t)-x(0))\right)+(\vec{v}, y)+\vec{u}\right) \exp \left\{i \lambda _ { 1 } ^ { - \frac { 1 } { 2 } } \left(v, x-x(0)-\frac{\dot{-}}{t}(x(t)-\right.\right. \\
& x(0)))\} d w_{\varphi}(x) d \vec{u} d \sigma(v) \\
= & \int_{L_{2}[0, t]} \exp \left\{i(v, y)+i\left(\zeta_{1}-\zeta_{0}\right) v\right\} \int_{\mathbb{R}^{r}} \int_{\mathbb{R}^{r}} \Phi(\lambda, \vec{u}) \Phi\left(\lambda_{1}, \vec{z}\right) D_{\lambda}(v) f_{r}((\vec{v}, y)+ \\
& \vec{u}+\vec{z}) d \vec{z} d \vec{u} d \sigma(v)
\end{aligned}
$$

by Lemma 2.1 and Theorem 3.2, where $\Phi$ and $B_{2}$ are given by (2.9) and (3.2), respectively, and $D_{\lambda}$ is as given in Theorem 3.3. Then we have by Lemma 3.1 and (3.3)

$$
\begin{aligned}
& K_{T_{\lambda}\left[\Psi \mid X_{0}\right]\left(\cdot, \xi_{0}\right)}^{\lambda_{1}}\left(y, \zeta_{0}\right) \\
= & \int_{L_{2}[0, t]} \exp \{i(v, y)\} \int_{\mathbb{R}^{r}} \int_{\mathbb{R}^{r}} \Phi(\lambda, \vec{u}) \Phi\left(\lambda_{1}, \vec{z}\right) D_{\lambda}(v) D_{\lambda_{1}}(v) f_{r}((\vec{v}, y)+ \\
& \vec{u}+\vec{z}) d \vec{z} d \vec{u} d \sigma(v)
\end{aligned}
$$

which holds for $\lambda_{1} \in \mathbb{C}_{+}$by the analytic continuation. Let $\vec{l}=\vec{u}+\vec{z}$. Then by (3.3) and the change of variable theorem

$$
\begin{aligned}
& T_{\bar{\lambda}}\left[T_{\lambda}\left[\Psi \mid X_{0}\right]\left(\cdot, \xi_{0}\right) \mid X_{0}\right]\left(\cdot, \zeta_{0}\right) \\
= & \left(\frac{|\lambda|^{2}}{4 \pi \operatorname{Re} \lambda}\right)^{\frac{r}{2}} \int_{L_{2}[0, t]} \exp \{i(v, y)\} \exp \left\{-\frac{t \operatorname{Re} \lambda}{|\lambda|^{2}} v^{2}\right\} \int_{\mathbb{R}^{r}} f_{r}((\vec{v}, y)-\vec{l}) \\
& \times \exp \left\{-\frac{|\lambda|^{2}}{4 \operatorname{Re} \lambda}\|\vec{l}\|_{\mathbb{R}^{r}}^{2}\right\} d \vec{l} d \sigma(v) \\
= & \epsilon^{-r}\left(f_{r} * \Phi(1, \cdot / \epsilon)\right)(\vec{v}, y) \int_{L_{2}[0, t]} \exp \{i(v, y)\} D_{1 / \epsilon^{2}}(v) d \sigma(v)
\end{aligned}
$$

where $\epsilon=\left(2 \operatorname{Re} \lambda /|\lambda|^{2}\right)^{1 / 2}>0$. Let $1 \leq p<\infty$. Then we have by Theorem 3.5 of [11] and the change of variable theorem

$$
\int_{C[0, t]}\left|T_{\bar{\lambda}}\left[T_{\lambda}\left[\Psi \mid X_{0}\right]\left(\cdot, \xi_{0}\right) \mid X_{0}\right]\left(y, \zeta_{0}\right)-\Psi(y)\right|^{p} d w_{\varphi}(y)
$$




$$
\begin{aligned}
\leq & \int_{\mathbb{R}^{r}}\left[\int_{L_{2}[0, t]}\left|\epsilon^{-r}\left(f_{r} * \Phi(1, \cdot / \epsilon)\right)(\vec{u}) D_{1 / \epsilon^{2}}(v)-f_{r}(\vec{u})\right| d|\sigma|(v)\right]^{p} d \vec{u} \\
\leq & \int_{\mathbb{R}^{r}}\left[\left|\epsilon^{-r}\left(f_{r} * \Phi(1, \cdot / \epsilon)\right)(\vec{u})-f_{r}(\vec{u})\right| \int_{L_{2}[0, t]} D_{1 / \epsilon^{2}}(v) d|\sigma|(v)+\right. \\
& \left.\left|f_{r}(\vec{u})\right| \int_{L_{2}[0, t]}\left|D_{1 / \epsilon^{2}}(v)-1\right| d|\sigma|(v)\right]^{p} d \vec{u}
\end{aligned}
$$

so that by the Minkowski inequality,

$$
\begin{aligned}
& \left\|T_{\bar{\lambda}}\left[T_{\lambda}\left[\Psi \mid X_{0}\right]\left(\cdot, \xi_{0}\right) \mid X_{0}\right]\left(\cdot, \zeta_{0}\right)-\Psi\right\|_{p} \\
\leq & \|\sigma\|\left\|\epsilon^{-r}\left(f_{r} * \Phi(1, \cdot / \epsilon)\right)-f_{r}\right\|_{p}+\left\|f_{r}\right\|_{p} \int_{L_{2}[0, t]}\left|D_{1 / \epsilon^{2}}(v)-1\right| d|\sigma|(v) .
\end{aligned}
$$

Letting $\lambda \rightarrow-i q$ through $\mathbb{C}_{+}$which satisfies $\epsilon \rightarrow 0$, we have the first part of the theorem by the dominated convergence theorem and Theorem 1.18 of [17]. Similarly, if $1 \leq p \leq \infty$, then we have the second part of the theorem by the dominated convergence theorem and Theorem 1.25 of $[17]$.

Using the same method as used in the proof of Theorem 3.2 in [5] with Theorems 2.4, 3.5, (2.4) and (3.5), we can prove the following theorems.

Theorem 4.3. Let $\Psi_{1}$ and $\Psi_{2}$ be as given in Theorem 2.4 and $q$ be a nonzero real number. Then for $\lambda \in \mathbb{C}_{+}$or $\lambda=-i q$, and $P_{X_{1}}$-a.e. $\left(\xi_{0}, \xi_{1}\right) \in \mathbb{R}^{2}$, we have the followings:

1. if $F_{r}, G_{r} \in \mathcal{A}_{r}^{(1)}$, then $\left[\left(\Psi_{1} * \Psi_{2}\right)_{\lambda} \mid X_{1}\right]\left(\cdot, \xi_{0}, \xi_{1}\right) \in L_{1}(C[0, t])$,

2. if $F_{r}, G_{r} \in \mathcal{A}_{r}^{(2)}$, then $\left[\left(\Psi_{1} * \Psi_{2}\right)_{\lambda} \mid X_{1}\right]\left(\cdot, \xi_{0}, \xi_{1}\right) \in L_{\infty}(C[0, t])$,

3. if $F_{r} \in \mathcal{A}_{r}^{(1)}$ and $G_{r} \in \mathcal{A}_{r}^{(2)}$, then $\left[\left(\Psi_{1} * \Psi_{2}\right)_{\lambda} \mid X_{1}\right]\left(\cdot, \xi_{0}, \xi_{1}\right) \in$ $L_{2}(C[0, t])$,

4. if $F_{r} \in \mathcal{A}_{r}^{(1)}$ and $G_{r} \in \mathcal{A}_{r}^{(1)} \cap \mathcal{A}_{r}^{(2)}$, then $\left[\left(\Psi_{1} * \Psi_{2}\right)_{\lambda} \mid X_{1}\right]\left(\cdot, \xi_{0}, \xi_{1}\right) \in$ $L_{1}(C[0, t]) \cap L_{2}(C[0, t])$, and

5. if $F_{r} \in \mathcal{A}_{r}^{(1)}$ and $G_{r} \in \mathcal{A}_{r}^{(\infty)}$, then $\left[\left(\Psi_{1} * \Psi_{2}\right)_{\lambda} \mid X_{1}\right]\left(\cdot, \xi_{0}, \xi_{1}\right) \in$ $L_{\infty}(C[0, t])$.

Theorem 4.4. If we replace $X_{1}$ by $X_{0}$ in Theorem 4.3, then the conclusions of the theorem are still true, where $\left[\left(\Psi_{1} * \Psi_{2}\right)_{\lambda} \mid X_{1}\right]\left(\cdot, \xi_{0}, \xi_{1}\right)$ is replaced by $\left[\left(\Psi_{1} * \Psi_{2}\right)_{\lambda} \mid X_{0}\right]\left(\cdot, \xi_{0}\right)$. 
Theorem 4.5. Under the assumptions as given in Theorem 2.4, we have for $\lambda \in \mathbb{C}_{+}, w_{\varphi}$-a.e. $y \in C[0, t]$ and $P_{X_{1}}$-a.e. $\left(\xi_{1}, \xi_{2}\right),\left(\zeta_{0}, \zeta_{1}\right) \in \mathbb{R}^{2}$

$$
\begin{aligned}
& T_{\lambda}\left[\left[\left(\Psi_{1} * \Psi_{2}\right)_{\lambda} \mid X_{1}\right]\left(\cdot, \xi_{0}, \xi_{1}\right) \mid X_{1}\right]\left(y, \zeta_{0}, \zeta_{1}\right) \\
= & {\left[T_{\lambda}\left[\Psi_{1} \mid X_{1}\right]\left(\frac{1}{\sqrt{2}} y, \frac{1}{\sqrt{2}}\left(\zeta_{0}+\xi_{0}\right), \frac{1}{\sqrt{2}}\left(\zeta_{1}+\xi_{1}\right)\right)\right] } \\
& \times\left[T_{\lambda}\left[\Psi_{2} \mid X_{1}\right]\left(\frac{1}{\sqrt{2}} y, \frac{1}{\sqrt{2}}\left(\zeta_{0}-\xi_{0}\right), \frac{1}{\sqrt{2}}\left(\zeta_{1}-\xi_{1}\right)\right)\right] .
\end{aligned}
$$

Proof. For $\lambda \in \mathbb{C}_{+}$and $\lambda_{1}>0$, we have by Lemma 2.1 and Theorem 2.4

$$
\begin{aligned}
& I_{\left[\left(\Psi_{1} * \Psi_{2}\right)_{\lambda} \mid X_{1}\right]\left(\cdot, \xi_{0}, \xi_{1}\right)}^{\lambda_{1}}\left(y, \zeta_{0}, \zeta_{1}\right) \\
= & \int_{L_{2}[0, t]} \int_{L_{2}[0, t]} H_{1}\left(\frac{1}{\sqrt{2}} y, \frac{1}{\sqrt{2}}\left(\zeta_{0}+\xi_{0}\right), \frac{1}{\sqrt{2}}\left(\zeta_{1}+\xi_{1}\right), v_{1}, v_{1}\right) H_{1}\left(\frac{1}{\sqrt{2}} y,\right. \\
& \left.\frac{1}{\sqrt{2}}\left(\zeta_{0}-\xi_{0}\right), \frac{1}{\sqrt{2}}\left(\zeta_{1}-\xi_{1}\right), v_{2}, v_{2}\right) \int_{C[0, t]} \exp \left\{i ( 2 \lambda _ { 1 } ) ^ { - \frac { 1 } { 2 } } \left(v_{1}+v_{2}, x-x(0)\right.\right. \\
& \left.\left.-\frac{\dot{t}}{t}(x(t)-x(0))\right)\right\} A_{2}\left(f_{r}, g_{r} ; 2 \lambda,\left(2 \lambda_{1}\right)^{-\frac{1}{2}}\left(\vec{v}, x-x(0)-\frac{\dot{-}}{t}(x(t)-x(0))\right)\right. \\
& \left.+\frac{1}{\sqrt{2}}(\vec{v}, y)+\frac{1}{\sqrt{2}}\left(\zeta_{1}-\zeta_{0}\right) \vec{V}_{t}, \frac{1}{\sqrt{2}} \xi_{0}, \frac{1}{\sqrt{2}} \xi_{1}, v_{1}-v_{2}\right) d w_{\varphi}(x) d \sigma_{1}\left(v_{1}\right) d \sigma_{2}\left(v_{2}\right) \\
= & \left(\frac{\lambda_{1}}{\pi}\right)^{\frac{r}{2}}\left(\frac{\lambda}{\pi}\right)^{\frac{r}{2}} \int_{L_{2}[0, t]} \int_{L_{2}[0, t]} H_{1}\left(\frac{1}{\sqrt{2}} y, \frac{1}{\sqrt{2}}\left(\zeta_{0}+\xi_{0}\right), \frac{1}{\sqrt{2}}\left(\zeta_{1}+\xi_{1}\right), v_{1}, v_{1}\right) \\
& \times H_{1}\left(\frac{1}{\sqrt{2}} y, \frac{1}{\sqrt{2}}\left(\zeta_{0}-\xi_{0}\right), \frac{1}{\sqrt{2}}\left(\zeta_{1}-\xi_{1}\right), v_{2}, v_{2}\right) \int_{\mathbb{R}^{r}} \int_{\mathbb{R}^{r}} f_{r}\left(\frac{1}{\sqrt{2}}(\vec{v}, y)+\frac{1}{\sqrt{2}}(\right. \\
& \left.\left.\zeta_{1}+\xi_{1}-\left(\zeta_{0}+\xi_{0}\right)\right) \vec{V}_{t}+T_{A}(\vec{z}+\vec{u})\right) g_{r}\left(\frac{1}{\sqrt{2}}(\vec{v}, y)+\frac{1}{\sqrt{2}}\left(\zeta_{1}-\xi_{1}-\left(\zeta_{0}-\xi_{0}\right)\right.\right. \\
& ) \vec{V}_{t}+T_{A}(\vec{z}-\vec{u})\right) A_{1}\left(2 \lambda, v_{1}-v_{2}, \vec{u}\right) A_{1}\left(2 \lambda_{1}, v_{1}+v_{2}, \vec{z}\right) d \vec{z} d \vec{u} d \sigma_{1}\left(v_{1}\right) d \sigma_{2}\left(v_{2}\right) .
\end{aligned}
$$

By the analytic continuation, we have the existence of $T_{\lambda_{1}}\left[\left[\left(\Psi_{1} * \Psi_{2}\right)_{\lambda} \mid X_{1}\right]\right.$ $\left.\left(\cdot, \xi_{0}, \xi_{1}\right) \mid X_{1}\right]\left(y, \zeta_{0}, \zeta_{1}\right)$ for $\lambda_{1} \in \mathbb{C}_{+}$. Let $\vec{w}=\vec{z}+\vec{u}$ and $\vec{l}=\vec{z}-\vec{u}$. Then for $\lambda \in \mathbb{C}_{+}$we have by the change of variable theorem

$$
\begin{aligned}
& T_{\lambda}\left[\left[\left(\Psi_{1} * \Psi_{2}\right)_{\lambda} \mid X_{1}\right]\left(\cdot, \xi_{0}, \xi_{1}\right) \mid X_{1}\right]\left(y, \zeta_{0}, \zeta_{1}\right) \\
= & \left(\frac{\lambda}{2 \pi}\right)^{r} \int_{L_{2}[0, t]} \int_{L_{2}[0, t]} H_{1}\left(\frac{1}{\sqrt{2}} y, \frac{1}{\sqrt{2}}\left(\zeta_{0}+\xi_{0}\right), \frac{1}{\sqrt{2}}\left(\zeta_{1}+\xi_{1}\right), v_{1}, v_{1}\right) H_{1}\left(\frac{1}{\sqrt{2}}\right. \\
& \left.\times y, \frac{1}{\sqrt{2}}\left(\zeta_{0}-\xi_{0}\right), \frac{1}{\sqrt{2}}\left(\zeta_{1}-\xi_{1}\right), v_{2}, v_{2}\right) \int_{\mathbb{R}^{r}} \int_{\mathbb{R}^{r}} f_{r}\left(\frac{1}{\sqrt{2}}(\vec{v}, y)+\frac{1}{\sqrt{2}}\left(\zeta_{1}+\xi_{1}\right.\right. \\
& \left.\left.-\left(\zeta_{0}+\xi_{0}\right)\right) \vec{V}_{t}+T_{A} \vec{w}\right) g_{r}\left(\frac{1}{\sqrt{2}}(\vec{v}, y)+\frac{1}{\sqrt{2}}\left(\zeta_{1}-\xi_{1}-\left(\zeta_{0}-\xi_{0}\right)\right) \vec{V}_{t}+T_{A} \vec{l}\right)
\end{aligned}
$$




$$
\times A_{1}\left(2 \lambda, v_{1}-v_{2}, \frac{1}{2}(\vec{w}-\vec{l})\right) A_{1}\left(2 \lambda, v_{1}+v_{2}, \frac{1}{2}(\vec{w}+\vec{l})\right) d \vec{w} d \vec{l} d \sigma_{1}\left(v_{1}\right) d \sigma_{2}\left(v_{2}\right) .
$$

By a calculation,

$$
\begin{aligned}
& A_{1}\left(2 \lambda, v_{1}-v_{2}, \frac{1}{2}(\vec{w}-\vec{l})\right) A_{1}\left(2 \lambda, v_{1}+v_{2}, \frac{1}{2}(\vec{w}+\vec{l})\right) \\
= & A_{1}\left(\lambda, v_{1}, \vec{w}\right) A_{1}\left(\lambda, v_{2}, \vec{l}\right)
\end{aligned}
$$

so that we have the theorem by Theorem 2.2 .

By Lemma 2.1, Theorems 2.2, 3.5 and the analytic continuation, we have the following theorem.

Theorem 4.6. Under the assumptions as given in Theorem 2.4, we have for $\lambda, \lambda_{1} \in \mathbb{C}_{+}$, $w_{\varphi}$-a.e. $y \in C[0, t], P_{X_{0}}$-a.e. $\xi_{0} \in \mathbb{R}$ and $P_{X_{1}}$-a.e. $\left(\zeta_{0}, \zeta_{1}\right) \in \mathbb{R}^{2}$

$$
\begin{aligned}
& T_{\lambda_{1}}\left[\left[\left(\Psi_{1} * \Psi_{2}\right)_{\lambda} \mid X_{0}\right]\left(\cdot, \xi_{0}\right) \mid X_{1}\right]\left(y, \zeta_{0}, \zeta_{1}\right) \\
= & \Gamma_{t}^{\frac{1}{2}}\left(\frac{\lambda}{\pi}\right)^{\frac{r}{2}}\left(\frac{\lambda_{1}}{\pi}\right)^{\frac{r}{2}} \int_{L_{2}[0, t]} \int_{L_{2}[0, t]} H_{1}\left(\frac{1}{\sqrt{2}} y, \frac{1}{\sqrt{2}} \zeta_{0}, \frac{1}{\sqrt{2}} \zeta_{1}, v_{1}+v_{2}, v_{1}+v_{2}\right) \\
& \times \int_{\mathbb{R}^{r}} \int_{\mathbb{R}^{r}} A_{1}\left(2 \lambda, v_{1}-v_{2}, \vec{u}\right) A_{1}\left(2 \lambda_{1}, v_{1}+v_{2}, \vec{z}\right) B_{1}\left(2 \lambda, v_{1}-v_{2}, \vec{u}\right) \\
& f_{r}\left(\frac{1}{\sqrt{2}}(\vec{v}, y)+\frac{1}{\sqrt{2}}\left(\zeta_{1}-\zeta_{0}\right) \vec{V}_{t}+T_{A}(\vec{u}+\vec{z})\right) g_{r}\left(\frac{1}{\sqrt{2}}(\vec{v}, y)+\frac{1}{\sqrt{2}}\left(\zeta_{1}-\right.\right. \\
& \left.\left.\zeta_{0}\right) \vec{V}_{t}-T_{A}(\vec{u}-\vec{z})\right) d \vec{z} d \vec{u} d \sigma_{1}\left(v_{1}\right) d \sigma_{2}\left(v_{2}\right)
\end{aligned}
$$

where $A_{1}, H_{1}$ and $B_{1}$ are given by (2.2), (2.8) and (3.1), respectively.

Theorem 4.7. Under the assumptions as given in Theorem 3.5, we have for $\lambda \in \mathbb{C}_{+}$, w $w_{\varphi}$-a.e. $y \in C[0, t]$ and $P_{X_{0}}$-a.e. $\xi_{0}, \zeta_{0} \in \mathbb{R}$

$$
\begin{aligned}
& T_{\lambda}\left[\left[\left(\Psi_{1} * \Psi_{2}\right)_{\lambda} \mid X_{0}\right]\left(\cdot, \xi_{0}\right) \mid X_{0}\right]\left(y, \zeta_{0}\right) \\
= & {\left[T_{\lambda}\left[\Psi_{1} \mid X_{0}\right]\left(\frac{1}{\sqrt{2}} y, 0\right)\right]\left[T_{\lambda}\left[\Psi_{2} \mid X_{0}\right]\left(\frac{1}{\sqrt{2}} y, 0\right)\right] . }
\end{aligned}
$$

Proof. Note that $B_{1}\left(2 \lambda_{1}, v_{1}+v_{2}, \vec{z}\right)=B_{1}\left(\lambda_{1}, \frac{1}{\sqrt{2}}\left(v_{1}+v_{2}\right), \vec{\alpha}\right)$ and $A_{1}\left(2 \lambda_{1}, v_{1}+v_{2}, \vec{z}\right)=A_{1}\left(\lambda_{1}, \frac{1}{\sqrt{2}}\left(v_{1}+v_{2}\right), \vec{\alpha}\right)$ if $\vec{\alpha}=\sqrt{2} \vec{z}$, where $A_{1}$ and $B_{1}$ are given by (2.2) and (3.1), respectively. For $\lambda \in \mathbb{C}_{+}$and $\lambda_{1}>0$, we 
have by Lemma 3.1, Theorem 4.6 and the change of variable theorem

$$
\begin{aligned}
& K_{\left[\left(\Psi_{1} * \Psi_{2}\right)_{\lambda} \mid X_{0}\right]\left(\cdot, \xi_{0}\right)}^{\lambda_{1}}\left(y, \zeta_{0}\right) \\
= & \Gamma_{t}^{\frac{1}{2}}\left(\frac{\lambda}{\pi}\right)^{\frac{r}{2}}\left(\frac{\lambda_{1}}{2 \pi}\right)^{\frac{r}{2}}\left(\frac{\lambda_{1}}{2 \pi t}\right)^{\frac{1}{2}} \int_{L_{2}[0, t]} \int_{L_{2}[0, t]} \exp \left\{\frac{i}{\sqrt{2}}\left(v_{1}+v_{2}, y\right)\right\} \int_{\mathbb{R}^{r}} A_{1}( \\
& \left.\left.2 \lambda, v_{1}-v_{2}, \vec{u}\right)\right) B_{1}\left(2 \lambda, v_{1}-v_{2}, \vec{u}\right) \int_{\mathbb{R}} \exp \left\{\frac{i}{\sqrt{2}} \frac{\zeta_{1}-\zeta_{0}}{t} \int_{0}^{t}\left(v_{1}(s)+v_{2}(s)\right) d s\right\} \\
& \times \int_{\mathbb{R}^{r}} A_{1}\left(\lambda_{1}, \frac{v_{1}+v_{2}}{2}, \vec{\alpha}\right) f_{r}\left(\frac{1}{\sqrt{2}}\left[(\vec{v}, y)+\left(\zeta_{1}-\zeta_{0}\right) \vec{V}_{t}+T_{A} \vec{\alpha}\right]+T_{A} \vec{u}\right) \\
& g_{r}\left(\frac{1}{\sqrt{2}}\left[(\vec{v}, y)+\left(\zeta_{1}-\zeta_{0}\right) \vec{V}_{t}+T_{A} \vec{\alpha}\right]-T_{A} \vec{u}\right) \exp \left\{-\frac{\lambda_{1}}{2} \frac{\left(\zeta_{1}-\zeta_{0}\right)^{2}}{t}\right\} \\
& d \vec{\alpha} d \zeta_{1} d \vec{u} d \sigma_{1}\left(v_{1}\right) d \sigma_{2}\left(v_{2}\right) \\
= & \Gamma_{t}\left(\frac{\lambda}{\pi}\right)^{\frac{r}{2}}\left(\frac{\lambda_{1}}{\pi}\right)^{\frac{r}{2}} \int_{L_{2}[0, t]} \int_{L_{2}[0, t]} \exp \left\{\frac{i}{\sqrt{2}}\left(v_{1}+v_{2}, y\right)\right\} \int_{\mathbb{R}^{r}} \int_{\mathbb{R}^{r}} A_{1}\left(2 \lambda, v_{1}-\right. \\
& \left.\left.v_{2}, \vec{u}\right)\right) B_{1}\left(2 \lambda, v_{1}-v_{2}, \vec{u}\right) A_{1}\left(2 \lambda_{1}, v_{1}+v_{2}, \vec{z}\right) B_{1}\left(2 \lambda_{1}, v_{1}+v_{2}, \vec{z}\right) f_{r}\left(\frac{1}{\sqrt{2}}(\vec{v}, y)\right. \\
& \left.+T_{A}(\vec{u}+\vec{z})\right) g_{r}\left(\frac{1}{\sqrt{2}}(\vec{v}, y)+T_{A}(\vec{z}-\vec{u})\right) d \vec{z} d \vec{u} d \sigma_{1}\left(v_{1}\right) d \sigma_{2}\left(v_{2}\right) .
\end{aligned}
$$

By the analytic continuation, we have the existence of $T_{\lambda_{1}}\left[\left[\left(\Psi_{1} * \Psi_{2}\right)_{\lambda} \mid X_{0}\right]\right.$ $\left.\left(\cdot, \xi_{0}\right) \mid X_{0}\right]\left(y, \zeta_{0}\right)$ for $\lambda_{1} \in \mathbb{C}_{+}$. Let $\vec{w}=\vec{z}+\vec{u}$ and $\vec{l}=\vec{z}-\vec{u}$. By a calculation, we can prove that for $\lambda \in \mathbb{C}_{+}, B_{1}\left(2 \lambda, v_{1}-v_{2}, \frac{1}{2}(\vec{w}-\vec{l})\right) B_{1}\left(2 \lambda, v_{1}+\right.$ $\left.v_{2}, \frac{1}{2}(\vec{w}+\vec{l})\right)=B_{1}\left(\lambda, v_{1}, \vec{w}\right) B_{1}\left(\lambda, v_{2}, \vec{l}\right)$ so that by the change of variable theorem

$$
\begin{aligned}
& T_{\lambda}\left[\left[\left(\Psi_{1} * \Psi_{2}\right)_{\lambda} \mid X_{0}\right]\left(\cdot, \xi_{0}\right) \mid X_{0}\right]\left(y, \zeta_{0}\right) \\
= & \Gamma_{t}\left(\frac{\lambda}{2 \pi}\right)^{r} \int_{L_{2}[0, t]} \int_{L_{2}[0, t]} \exp \left\{\frac{i}{\sqrt{2}}\left(v_{1}, y\right)\right\} \exp \left\{\frac{i}{\sqrt{2}}\left(v_{2}, y\right)\right\} \int_{\mathbb{R}^{r}} \int_{\mathbb{R}^{r}} A_{1}(\lambda, \\
& \left.v_{1}, \vec{w}\right) A_{1}\left(\lambda, v_{2}, \vec{l}\right) B_{1}\left(\lambda, v_{1}, \vec{w}\right) B_{1}\left(\lambda, v_{2}, \vec{l}\right) f_{r}\left(\frac{1}{\sqrt{2}}(\vec{v}, y)+T_{A} \vec{w}\right) g_{r}\left(\frac{1}{\sqrt{2}}(\vec{v},\right. \\
& \left.y)+T_{A} \vec{l}\right) d \vec{w} d \vec{l} d \sigma_{1}\left(v_{1}\right) d \sigma_{2}\left(v_{2}\right)
\end{aligned}
$$

which completes the proof by Theorem 3.2.

Finally, we have the following relationships from Theorems 2.2, 2.4, $3.2,3.5,4.3,4.4,4.5$ and 4.7 .

Theorem 4.8. Let $\Psi_{1}$ and $\Psi_{2}$ be as given in Theorem 2.4 and $q$ be a nonzero real number. Then we have the followings: 
1. if $F_{r}, G_{r} \in \mathcal{A}_{r}^{(1)}$, then we have for $w_{\varphi}$-a.e. $y \in C[0, t]$ and $P_{X_{1}}$-a.e. $\left(\xi_{0}, \xi_{1}\right),\left(\zeta_{0}, \zeta_{1}\right) \in \mathbb{R}^{2}$

$$
\begin{aligned}
& T_{q}^{(1)}\left[\left[\left(\Psi_{1} * \Psi_{2}\right)_{q} \mid X_{1}\right]\left(\cdot, \xi_{0}, \xi_{1}\right) \mid X_{1}\right]\left(y, \zeta_{0}, \zeta_{1}\right) \\
= & {\left[T_{q}^{(1)}\left[\Psi_{1} \mid X_{1}\right]\left(\frac{1}{\sqrt{2}} y, \frac{1}{\sqrt{2}}\left(\zeta_{0}+\xi_{0}\right), \frac{1}{\sqrt{2}}\left(\zeta_{1}+\xi_{1}\right)\right)\right] } \\
& \times\left[T_{q}^{(1)}\left[\Psi_{2} \mid X_{1}\right]\left(\frac{1}{\sqrt{2}} y, \frac{1}{\sqrt{2}}\left(\zeta_{0}-\xi_{0}\right), \frac{1}{\sqrt{2}}\left(\zeta_{1}-\xi_{1}\right)\right)\right],
\end{aligned}
$$

and for $P_{X_{0}}$-a.e. $\xi_{0}, \zeta_{0} \in \mathbb{R}$

$$
\begin{aligned}
& T_{q}^{(1)}\left[\left[\left(\Psi_{1} * \Psi_{2}\right)_{q} \mid X_{0}\right]\left(\cdot, \xi_{0}\right) \mid X_{0}\right]\left(y, \zeta_{0}\right) \\
= & {\left[T_{q}^{(1)}\left[\Psi_{1} \mid X_{0}\right]\left(\frac{1}{\sqrt{2}} y, 0\right)\right]\left[T_{q}^{(1)}\left[\Psi_{2} \mid X_{0}\right]\left(\frac{1}{\sqrt{2}} y, 0\right)\right], }
\end{aligned}
$$

2. if $F_{r} \in \mathcal{A}_{r}^{(1)}$ and $G_{r} \in \mathcal{A}_{r}^{(2)}$, then we have for $w_{\varphi}$-a.e. $y \in C[0, t]$ and $P_{X_{1}}$-a.e. $\left(\xi_{0}, \xi_{1}\right),\left(\zeta_{0}, \zeta_{1}\right) \in \mathbb{R}^{2}$

$$
\begin{aligned}
& T_{q}^{(2)}\left[\left[\left(\Psi_{1} * \Psi_{2}\right)_{q} \mid X_{1}\right]\left(\cdot, \xi_{0}, \xi_{1}\right) \mid X_{1}\right]\left(y, \zeta_{0}, \zeta_{1}\right) \\
= & {\left[T_{q}^{(1)}\left[\Psi_{1} \mid X_{1}\right]\left(\frac{1}{\sqrt{2}} y, \frac{1}{\sqrt{2}}\left(\zeta_{0}+\xi_{0}\right), \frac{1}{\sqrt{2}}\left(\zeta_{1}+\xi_{1}\right)\right)\right] } \\
& \times\left[T_{q}^{(2)}\left[\Psi_{2} \mid X_{1}\right]\left(\frac{1}{\sqrt{2}} y, \frac{1}{\sqrt{2}}\left(\zeta_{0}-\xi_{0}\right), \frac{1}{\sqrt{2}}\left(\zeta_{1}-\xi_{1}\right)\right)\right]
\end{aligned}
$$

and for $P_{X_{0}}$-a.e. $\xi_{0}, \zeta_{0} \in \mathbb{R}$

$$
\begin{aligned}
& T_{q}^{(2)}\left[\left[\left(\Psi_{1} * \Psi_{2}\right)_{q} \mid X_{0}\right]\left(\cdot, \xi_{0}\right) \mid X_{0}\right]\left(y, \zeta_{0}\right) \\
= & {\left[T_{q}^{(1)}\left[\Psi_{1} \mid X_{0}\right]\left(\frac{1}{\sqrt{2}} y, 0\right)\right]\left[T_{q}^{(2)}\left[\Psi_{2} \mid X_{0}\right]\left(\frac{1}{\sqrt{2}} y, 0\right)\right] . }
\end{aligned}
$$

\section{References}

[1] Cameron R.H., Storvick D.A., Some Banach algebras of analytic Feynman integrable functionals, Lecture Notes in Mathematics, 798, Springer, Berlin-New York, 1980

[2] Chang S.J., Skoug D., The effect of drift on conditional Fourier-Feynman transforms and conditional convolution products, Int. J. Appl. Math., 2000, 2(4), $505-527$

[3] Cho D.H., Conditional Fourier-Feynman transforms and convolutions of unbounded functions on a generalized Wiener space, preprint

[4] Cho D.H., A time-independent conditional Fourier-Feynman transform and convolution product on an analogue of Wiener space, Bull. Korean Math. Soc., (in Press, in Korea) 
[5] Cho D.H., A time-dependent conditional Fourier-Feynman transform and convolution product on an analogue of Wiener space, preprint

[6] Cho D.H., Conditional integral transforms and convolutions of bounded functions on an analogue of Wiener space, preprint

[7] Cho D.H., Conditional integral transforms and conditional convolution products on a function space, Integral Transforms Spec. Funct., 2012, 23(6), 405-420

[8] Cho D.H., A simple formula for an analogue of conditional Wiener integrals and its applications II, Czechoslovak Math. J., 2009, 59(2), 431-452

[9] Cho D.H., A simple formula for an analogue of conditional Wiener integrals and its applications, Trans. Amer. Math. Soc., 2008, 360(7), 3795-3811

[10] Cho D.H., Kim B.J., Yoo I., Analogues of conditional Wiener integrals and their change of scale transformations on a function space, J. Math. Anal. Appl., 2009, $359(2), 421-438$

[11] Im M.K., Ryu K.S., An analogue of Wiener measure and its applications, J. Korean Math. Soc., 2002, 39(5), 801-819

[12] Johnson G.W., Skoug D.L., The Cameron-Storvick function space integral: an $\mathcal{L}\left(L_{p}, L_{p^{\prime}}\right)$ theory, Nagoya Math. J., 1976, 60, 93-137

[13] Kim M.J., Conditional Fourier-Feynman transform and convolution product on a function space, Int. J. Math. Anal., 2009, 3(10), 457-471

[14] Park S.B., Cho D.H., Choi Y.H., Conditional Fourier-Feynman transforms and convolutions over continuous paths, Int. Math. Forum. 2013, 8(9), 443-456

[15] Ryu K.S., Im M.K., A measure-valued analogue of Wiener measure and the measure-valued Feynman-Kac formula, Trans. Amer. Math. Soc., 2002, 354(12), 4921-4951

[16] Ryu K.S., Im M.K., Choi K.S., Survey of the theories for analogue of Wiener measure space, Interdiscip. Inform. Sci., 2009, 15(3), 319-337

[17] Stein E.M., Weiss G., Introduction to Fourier Analysis on Euclidean Spaces, Princeton Univ. Press, Princeton, 1971.

\author{
Dong Hyun Cho \\ Department of Mathematics, Kyonggi University, \\ Suwon 443-760, Korea. \\ E-mail: j94385@kyonggi.ac.kr
}

